\title{
Distribution of waves in the buildings and constructions having regular structure
}

\author{
Vladimir Mondrus ${ }^{1, *}$, and Stanislav Shutovskiy ${ }^{1}$ \\ ${ }^{1}$ NRU MGSU, Editorial Department, 129337, 26, Yaroslavskoye Shosse, Moscow, Russia
}

\begin{abstract}
In article settlement models of the buildings and constructions having periodic (regular) structure with a masses concentrated in nods are considered. Passing of waves in such systems is considered. For a special case the picture of lines of equal frequency allowing to determine limit frequencies of waves which can pass through this model is constructed.
\end{abstract}

Article is devoted to research of settlement models of the buildings and constructions representing themselves periodic (regular) systems with a masses concentrated in nods. Further we assume that interaction takes place only between the next masses. In the most general view the three-dimensional settlement model of the building is shown in Fig. 1.

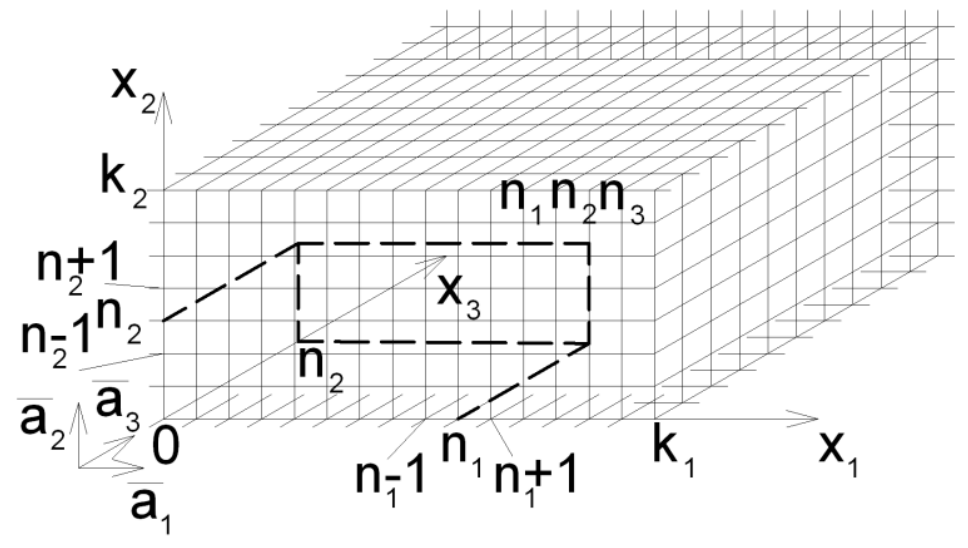

Fig. 1. Three-dimensional general model of the building

Let's consider the three-dimensional periodic orthogonal settlement model presented in Fig. 1. We believe that elements communications between masses, parallel to various axes of coordinates, possess various stiffness characteristics, and elements of one direction have various stiffness characteristics depending on the direction of mutual forward shift of masses[1].

* Corresponding author: mondrus@mail.ru 
From all $\alpha\left(\alpha=x_{1}, x_{2}, x_{3}\right)$ the values of size, possible for communication of any one direction, $\alpha\left(\alpha=x_{1}, x_{2}, x_{3}\right) E_{\Pi \alpha \beta}\left(n n^{\prime}\right)_{\text {determined by a matrix }}$

$$
\left\|\begin{array}{ccc}
\alpha & \alpha & \alpha \\
E_{\Pi 11}\left(n n^{\prime}\right) & E_{\Pi 12}\left(n n^{\prime}\right) & E_{\Pi 13}\left(n n^{\prime}\right) \\
\alpha & \alpha & \alpha \\
E_{\Pi 21}\left(n n^{\prime}\right) & E_{\Pi 22}\left(n n^{\prime}\right) & E_{\Pi 23}\left(n n^{\prime}\right) \\
\alpha & \alpha & E^{\alpha} \\
E_{\Pi 31}\left(n n^{\prime}\right) & E_{\Pi 32}\left(n n^{\prime}\right) & E_{\Pi 33}\left(n n^{\prime}\right)
\end{array}\right\|
$$

let's consider only three, standing on the main diagonal and making real physical sense for settlement models of buildings. Characteristics of connections,

which axis is parallel to an axis $x_{1}$ :

$$
E_{\Pi 11}\left(\stackrel{\alpha}{n n^{\prime}}\right)=\gamma_{11} ; \quad E_{\Pi 12}\left(\stackrel{\alpha}{n n^{\prime}}\right)=\gamma_{12} ; \quad E_{\Pi 13}\left(\stackrel{\alpha}{n n^{\prime}}\right)=\gamma_{13}
$$

which axis is parallel to an axis $x_{2}$ :

$$
E_{\Pi 21}\left(\stackrel{\alpha}{n n^{\prime}}\right)=\gamma_{21} ; \quad E_{\Pi 22} \stackrel{\alpha}{\left(n n^{\prime}\right)}=\gamma_{22} ; \quad E_{\Pi 23} \stackrel{\alpha}{\left(n n^{\prime}\right)}=\gamma_{23}
$$

which axis is parallel to an axis $x_{3}$ :

$$
E_{\Pi 31} \stackrel{\alpha}{\left(n n^{\prime}\right)}=\gamma_{31} ; \quad E_{\Pi 32} \stackrel{\alpha}{\alpha}\left(n n^{\prime}\right)=\gamma_{32} ; \quad E_{\Pi 33}\left(\stackrel{\alpha}{n n^{\prime}}\right)=\gamma_{33}
$$

Other of possible values of size $E_{\Pi \alpha \beta}\left(\begin{array}{c}\alpha \\ n\end{array}\right)$ it is believed equal to zero, as not making physical sense.

Thus sizes $\gamma_{\alpha \beta}$ characterize rigidityof connections which axis is parallel to an axis of coordinates $\alpha$ at mutual shift of the connected masses along the axis parallel to an axis of coordinates $\beta$ (Fig. 2) 


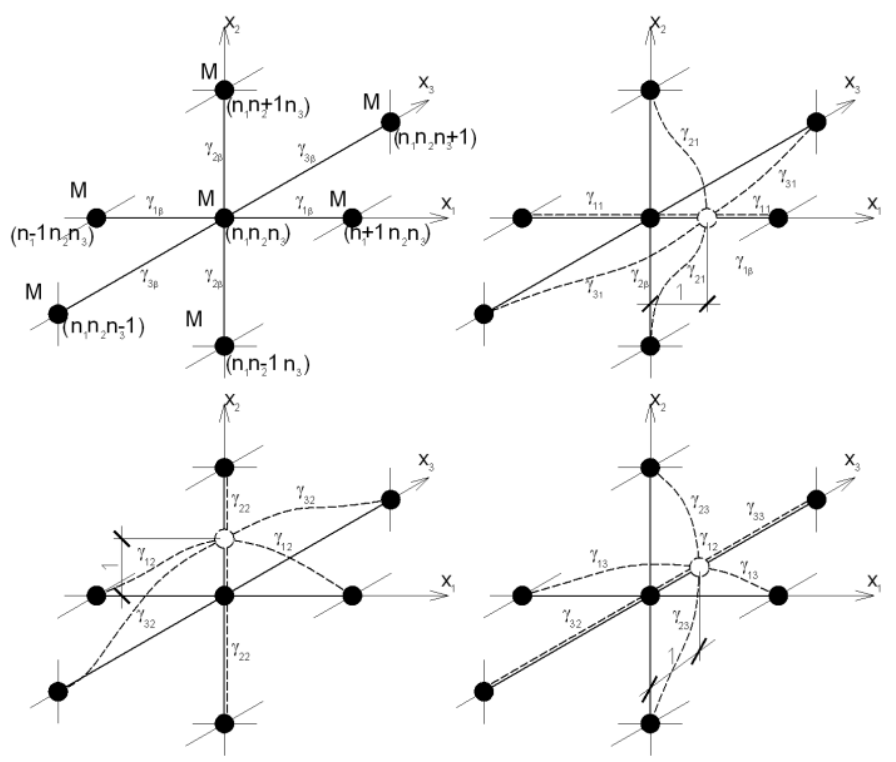

Fig. 2. Definition of stiffness of connections

Besides forward movements of masses also their turns round the axes parallel to axes of coordinates are possible. Such connections are designated as $\gamma_{\alpha \beta}^{*}$.

It is possible to write down the movement equation for $\alpha$ - any mass of settlement model which is working out shifts[2]. At $\alpha=x_{1}$ the equation will register in a look

$$
M \ddot{u}\left(n_{1} n_{2}^{x_{1}} n_{3}\right)=\gamma_{11} \Delta_{n 1} u\left(n_{1} n_{2} n_{3}\right)+\gamma_{21} \Delta_{n 2} u\left(n_{1} n_{2} n_{3}\right)+\gamma_{31} \Delta_{n 3} u\left(n_{1} n_{2} n_{3}\right)
$$

where

$$
\begin{gathered}
\Delta_{n 1} u\left(n_{1} n_{2} n_{3}\right)=u\left(n_{1}+1_{1}^{x_{1}}, n_{2}, n_{3}\right)-2 u\left(n_{1} n_{2} n_{3}\right)+u\left(n_{1}-1_{1}^{x_{1}} n_{2}, n_{3}\right) \\
\Delta_{n 2} u\left(n_{1} n_{2} n_{3}\right)=u\left(n_{1}, n_{2}^{x_{1}}+1, n_{3}\right)-2 u\left(n_{1} n_{2} n_{3}\right)+u\left(n_{1}, n_{2}^{x_{1}}-1, n_{3}\right) \\
\Delta_{n 3} u\left(n_{1} n_{2} n_{3}\right)=u\left(n_{1}, n_{2}, n_{3}+1\right)-2 u\left(n_{1} n_{2} n_{3}\right)+u\left(n_{1}-1, n_{2}, n_{3}-1\right)
\end{gathered}
$$

Solving this equation we find:

$$
\frac{M \omega^{2}}{4}=\gamma_{11} \sin ^{2}\left(\pi \bar{c} \bar{a}_{1}\right)+\gamma_{21} \sin ^{2}\left(\pi \bar{c} \bar{a}_{2}\right)+\gamma_{31} \sin ^{2}\left(\pi \bar{c} \bar{a}_{3}\right)
$$

Having divided both parts of equality (7) on $\gamma_{11}$, we will receive expression in dimensionless coordinates 


$$
\Gamma_{1}=\sin ^{2}\left(\pi \bar{c} \bar{a}_{1}\right)+\chi_{1} \sin ^{2}\left(\pi \bar{c} \bar{a}_{2}\right)+\chi_{2} \sin ^{2}\left(\pi \bar{c} \bar{a}_{3}\right)
$$

where

$$
\Gamma_{1}=\frac{M \omega^{2}}{4 \gamma_{11}} \chi_{1}=\frac{\gamma_{21}}{\gamma_{11}} \chi_{2}=\frac{\gamma_{31}}{\gamma_{11}}
$$

- dimensionless parameters.

Equality (8) is a dispersive ratio for one of three branches of a spectrum of threedimensional settlement model, the corresponding component of progress of masses along an axis X1.

Similarly dispersive ratios for other branches of a spectrum (turn out at forward and the movements of mass of settlement model and their turn round axes).

Let's consider rectangular two-dimensional settlement model - see Fig. 3.
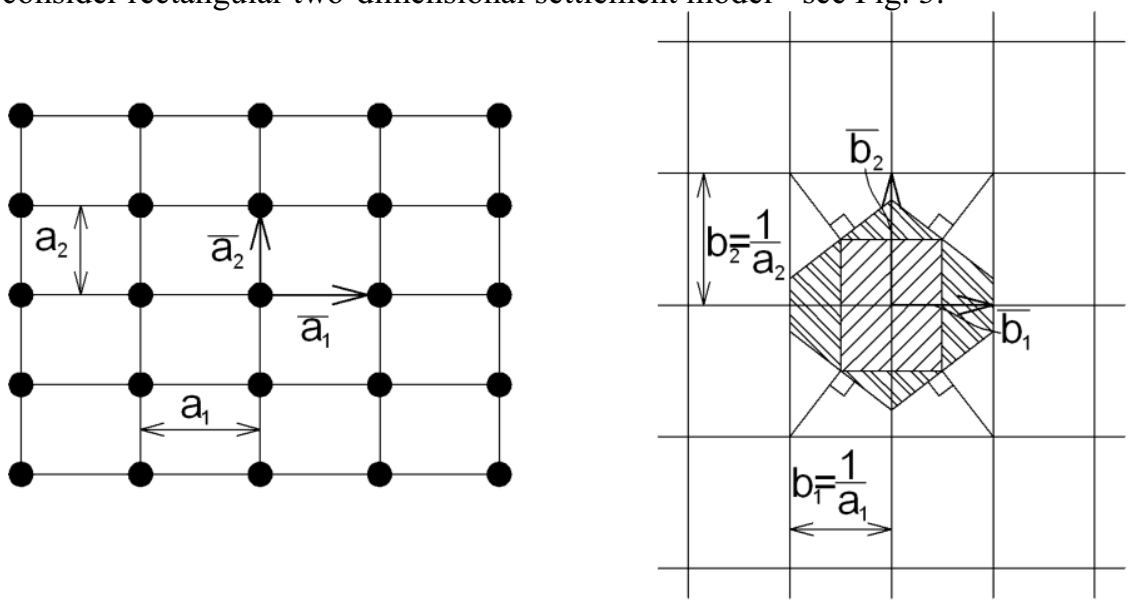

Fig. 3. Two-dimensional lattice of flat settlement model
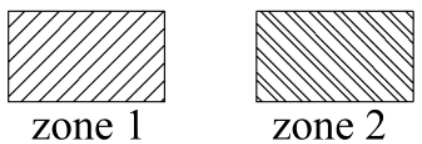

For creation of the return lattice in a direct lattice the distance $\bar{a}_{\alpha}=\left|\bar{a}_{\alpha}\right|$ between parallel ranks of masses is defined $\bar{a}_{\alpha}=\left|\bar{a}_{\alpha}\right|$. Having carried out a piece $\quad \bar{a}_{\alpha} \quad \frac{1}{\left|\bar{a}_{\alpha}\right|}$ of the direction, perpendicular to the corresponding ranks, we receive a vector of the return lattice. For the considered lattice straight line the return lattice will also be rectangular. The structure of the two first zones of the return lattice is represented in Fig. 3. For two-dimensional settlement model function $v(\bar{c})$ or $\omega(\bar{c})$ depends on variables $c_{1}$ and $c_{2}$ (a vector component $\bar{c}$ ) and on border of the first zone its normal derivative has to address in zero. Using dispersive ratios for two-dimensional 
settlement models, in the plane $c_{1} c_{2}$ it is possible to construct family of curves, along each of which frequency is constant. For such lines of equal frequency B. P. Wolfson entered the name "izosikhnotit". All family of curves characterizing change of frequency of dependence from $\bar{c}$ is called as a picture izosikhnotit[3]. The example of a picture izosikhnotit for the two-dimensional settlement model represented in Fig. 3, it is provided on Fig. 4.
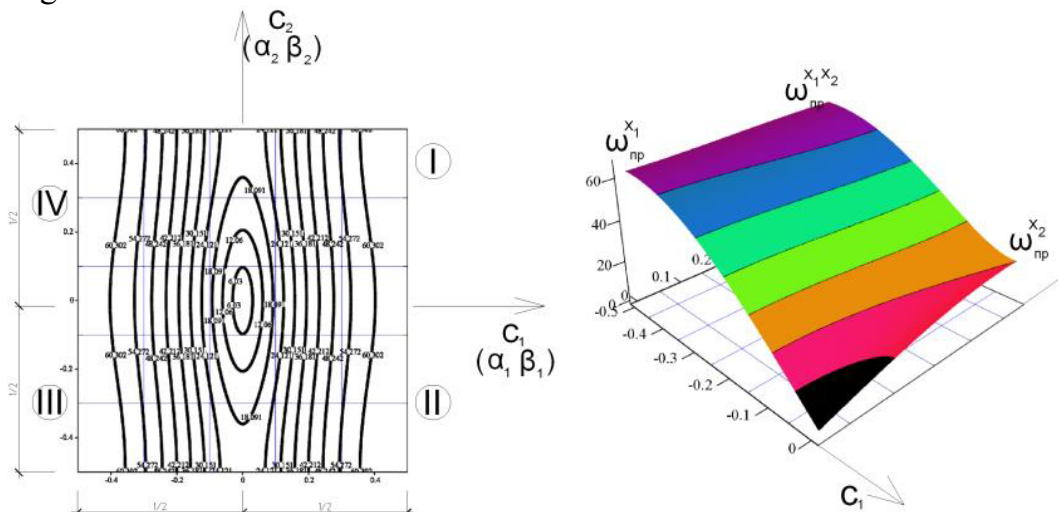

$\mathrm{C}_{2}$

Fig. 4. The picture "izosikhnotit" (lines of equal frequency)

It is possible to consider a concrete dispersive ratio for two-dimensional settlement model. For example,

$$
\Gamma_{1}=\sin ^{2}\left(\pi \bar{c} \bar{a}_{1}\right)+\chi_{1} \sin ^{2}\left(\pi \bar{c} \bar{a}_{2}\right)
$$

where

$$
\Gamma_{1}=\frac{M \omega^{2}}{4 \gamma_{11}}, \chi_{1}=\frac{\gamma_{21}}{\gamma_{11}}
$$

This dispersive ratio corresponds to one of branches of a spectrum of two-dimensional model. We will be set by concrete sizes of parameters:

$$
M=\frac{20000 \mathrm{~kg}}{980 \mathrm{~cm} / \mathrm{s}^{2}} \approx 20 \frac{\mathrm{kg} \cdot \mathrm{s}^{2}}{\mathrm{~cm}} ; c_{1}=360 \mathrm{~cm} ; c_{2}=270 \mathrm{~cm} ; \gamma_{11}=2 \cdot 10^{4} \frac{\mathrm{kg}}{\mathrm{cm}^{2}} ; \gamma_{21}=2 \cdot 10^{3} \frac{\mathrm{kg}}{\mathrm{cm}^{2}}
$$

At such values of parameters the picture izosikhnotit $\omega(\bar{c})$, constructed in the first zone of the return lattice, has the appearance shown in Fig. 4. The ratio for determination of frequency thus registers in a look:

$$
\omega^{2}=400\left(10 \sin ^{2} \pi c_{1}+\sin ^{2} \pi c_{2}\right)
$$

Follows from the ratios given above that frequency $\omega$ - size significantly positive and is the periodic function of coordinates $c_{\alpha}$ of a wave vector $\bar{c}_{\text {symmetric relatively, axes of }}$ coordinates, having an absolute maximum in points $c_{1}= \pm 1 / 2, c_{2}= \pm 1 / 2$ and 
stationary saddle points with coordinates $c_{1}= \pm 1 / 2, c_{2}=0$ and $c_{1}=0 c_{2}= \pm 1 / 2$. The period, functions in the direction of both axes of coordinates is equal 1 , as

$$
\Gamma_{1}=\sin ^{2} \pi\left(c_{1}+1\right)+\chi_{1} \sin ^{2} \pi\left(c_{2}+1\right)=\sin ^{2} \pi c_{1}+\chi_{1} \sin ^{2} \pi c_{2}
$$

Existence of special points specifies that for any direction of distribution of a wave for discrete periodic settlement models there are limit frequencies which we will call further characteristic. They are defined from a ratio (7):

$$
\omega_{n p}^{x_{1}}=2 \sqrt{\frac{\gamma_{11}}{M}} c_{1}=\frac{1}{2}{ }_{()} c_{2}=0
$$

- local limit frequency for the direction of the wave vector coinciding with an axis $O x_{1}$;

$$
\omega_{n p}^{x_{2}}=2 \sqrt{\frac{\gamma_{11}}{M}} c_{1}=0{ }_{()} c_{2}=\frac{1}{2}
$$

- local limit frequency for the direction of the wave vector coinciding with an axis $O x_{2}$

$$
\left.\omega_{n p}^{x_{1} x_{2}}=2 \sqrt{\frac{\gamma_{11}+\gamma_{21}}{M}}, c_{1}=c_{2}=\frac{1}{2}\right)
$$

- absolute limit frequency of settlement model for the direction of the wave vector coinciding with a diagonal of any of zone quadrants. If the frequency of the extending wave is more or is equal $\omega_{n p}^{x_{1} x_{2}}$, irrespective of the direction of distribution amplitude of this wave at its movement in settlement model will decrease from weight to weight.

The spatial discrete settlement model of the building or construction generally can have 6 branches of a spectrum of own frequencies corresponding to the 3rd to components of progress and the 3rd possible turns of masses. The dispersive ratio testifies to the certain restrictions imposed by various factors on possibility of distribution in settlement model of the corresponding wave mode (fluctuation)[4]. These restrictions, are generally connected with that for any direction of a wave vector of the wave extending in the building there is quite certain pass-band limited to limit frequencies. Fluctuations with the frequencies which are not getting to a pass-band practically in the building cannot extend. Thus, if to the building (construction) the wave of the general type capable to cause all six possible types of fluctuations comes, that, depending on characteristics of the falling wave and design of the building in the last waves of one types can actively extend and practically waves of other types cannot extend.

In real buildings and constructions the same seismic wave in standing nearby on a surface of the earth and buildings and constructions, various on the constructive decision, can cause fluctuations of absolutely different types (vertical, horizontal, tortional). The same wave in the constructions, identical on the constructive decision located under different corners to the front of a wave can or cause active fluctuations, or not cause almost any fluctuations. Similar results obviously have to take place at impact on buildings and constructions of a blast wave, a wind, etc. 
Depending on parameters of a wave and characteristics of a construction it can appear that the wave from a source in a construction mainly extends only in any chosen directions lying in certain space angles - for three-dimensional settlement models or certain flat corners - for two-dimensional settlement models. In special cases the wave can extend only in the direction of some beams proceeding from a construction point in which the source is located. Thus in certain directions mainly extends waves of one type. Along with the specified special cases perhaps also that the wave extends in all directions from a source without attenuation, or, on the contrary, in all directions actively fades.

\section{References}

1. L.Brilluen, M.Parody, Distribution of waves in periodic structures. (SILT, Moscow, 1959)

2. B. P. Wolfson, Researches on the theory of constructions, under. edition of the prof. B. G. Korenev, member correspondent of Academy of Sciences of the USSR I. M. Rabinovich, prof. A. F. Smirnov, XYII (1969).

3. B. P. Wolfson, Researches on the theory of constructions, under. edition of the prof. B. G. Korenev, member correspondent of Academy of Sciences of the USSR I. M. Rabinovich, prof. A. F. Smirnov, XIX (1972).

4. M. A. Dashevsky, Works TsNIISK of V. A. Kucherenko, 43 (1975). 